\title{
GENERALIZED SYMMETRIC FUNCTIONS AND INVARIANTS OF MATRICES
}

\author{
FRANCESCO VACCARINO
}

\begin{abstract}
It is well known that over an infinite field the ring of symmetric functions in a finite number of variables is isomorphic to the one of polynomial functions on a single matrix that are invariants by the action of conjugation by general linear group. We generalize this result showing that the abelianization of the algebra of the symmetric tensors of fixed order over a free associative algebra is isomorphic to the algebra of the polynomials invariants of several matrices over an infinite field or the integers. While proving the main result we find generators and relations of abelianized divided powers and symmetric products over any commutative ring.
\end{abstract}

\section{INTRODUCTION}

Let $\mathbb{K}$ be an infinite field and let $\mathbb{K}\left[y_{1}, \ldots, y_{n}\right]^{S_{n}}$ be the the ring of symmetric polynomials in $n$ variables. The general linear group $G L(n, \mathbb{K})$ acts by conjugation on the full ring $\operatorname{Mat}(n, \mathbb{K})$ of $n \times n$ matrices over $\mathbb{K}$. Denote by $\mathbb{K}[\operatorname{Mat}(n, \mathbb{K})]^{G L(n, \mathbb{K})}$ the ring of the polynomial invariants for this actions. It is well known that

$$
\mathbb{K}[\operatorname{Mat}(n, \mathbb{K})]^{G L(n, \mathbb{K})} \cong \mathbb{K}\left[y_{1}, \ldots, y_{n}\right]^{S_{n}} .
$$

Let $M$ be a $\mathbb{K}$-module. Consider the tensor product $T_{\mathbb{K}}^{n}(M):=M^{\otimes n}$, the symmetric group acts on $T_{\mathbb{K}}^{n}(M)$ as a group of $\mathbb{K}$-module automorphisms and we denote by $T S_{\mathbb{K}}^{n}(M):=\left(M^{\otimes n}\right)^{S_{n}}$ the sub- $\mathbb{K}$-module of the invariants for this action. The elements of $T S_{\mathbb{K}}^{n}(M)$ are called the symmetric tensors of order $n$. If $M$ is a $\mathbb{K}$-algebra then $T S_{\mathbb{K}}^{n}(M)$ is a sub- $\mathbb{K}$-algebra of $M$.

Let $F:=\mathbb{K}\left\{x_{1}, \ldots, x_{m}\right\}$ be a free associative non commutative algebra on $m$ variables. For a $\mathbb{K}$-algebra $A$ we write $A^{a b}:=A /[A, A]$ for the abelianization of $A$, where $[A, A]$ denotes the ideal generated by the commutators. Consider

(i) $T S_{\mathbb{K}}^{n}(F)$ and

(ii) $T S_{\mathbb{K}}^{n}(F)^{a b}$ the abelianization of $T S_{\mathbb{K}}^{n}(F)$

Note that when $m=1$ then $F$ is commutative and $T S_{\mathbb{K}}^{n}(F)=T S_{\mathbb{K}}^{n}(F)^{a b}$.

We will find generators for both $T S_{\mathbb{K}}^{n}(F), T S_{\mathbb{K}}^{n}(F)^{a b}$ and for divided powers of degree $n$ over any algebra. The result presented are refinement of the one due to Ziplies [18] and are proven in a rather simple way.

We also find relations, the first syzygy, for $T S_{\mathbb{K}}^{n}(F)^{a b}$ and then for the abelianization of divided powers of any algebra and for divided powers of any commutative algebra.

1991 Mathematics Subject Classification. Primary 05E05, 16G99 ; Secondary 13A50.

Key words and phrases. Symmetric functions, invariants of matrices, divided powers.

The author is supported by research grant Politecnico di Torino n.119 - 2004. 
Using the presentation we found we then prove the following generalization of the isomorphism (1.1).

Theorem 1.1. Let $\mathbb{K}$ be an infinite field or the ring of integers and let the general linear group $G L(n, \mathbb{K})$ acts by simultaneous conjugation on $m$ copies of $\operatorname{Mat}(n, \mathbb{K})$. Denote by $\mathbb{K}\left[M a t(n, \mathbb{K})^{m}\right]^{G L(n, \mathbb{K})}$ the ring of the invariants for this actions. Then

$$
\mathbb{K}\left[\operatorname{Mat}(n, \mathbb{K})^{m}\right]^{G L(n, \mathbb{K})} \cong T S_{\mathbb{K}}^{n}\left(\mathbb{K}\left\{x_{1}, \ldots, x_{m}\right\}\right)^{a b}
$$

Remark 1.2. Let $\mathbb{K}$ be an infinite field, in [16 we proved that there is an isomorphism $T S_{\mathbb{K}}^{n}\left(\mathbb{K}\left[x_{1}, \ldots, x_{m}\right]\right) \cong \mathbb{K}\left[Z_{n, \text { red }}^{m}\right]^{G L(n, \mathbb{K})}$ where $Z_{n, \text { red }}^{m}$ is the variety of $m$-tuples of commuting $n \times n$ matrices. When char $\mathbb{K}=0$ then we showed that the above isomorphism extends to the affine schemes i.e. $T S_{\mathbb{K}}^{n}\left(\mathbb{K}\left[x_{1}, \ldots, x_{m}\right]\right) \cong$ $\mathbb{K}\left[Z_{n}^{m}\right]^{G L(n, \mathbb{K})}$, where $Z_{n}^{m}$ is the affine scheme of $m$-tuples of commuting $n \times n$ matrices. Some links with the Hilbert scheme of points will be given at ICM 2006 17

\section{Conventions And notation}

All rings or algebras over a commutative ring will be with identity. We denote by $\mathbb{N}$ the natural numbers. Let $\mathbb{K}$ be a commutative ring. We will use the following categories

- $\mathcal{N}, \mathcal{N}_{\mathbb{K}}$ the categories of non commutative rings and non commutative $\mathbb{K}$ algebras

- $\mathcal{C}, \mathcal{C}_{\mathbb{K}}$ the categories of commutative rings and commutative $\mathbb{K}$-algebras

- $M o d_{\mathbb{K}}$ for the category of $\mathbb{K}$-modules

- Set the category of sets

- We set $\mathcal{A}(B, C):=\operatorname{Hom}_{\mathcal{A}}(B, C)$ in a category $\mathcal{A}$ with $B, C \in O b(\mathcal{A})$ objects in $\mathcal{A}$.

- For $A$ a set and any additive monoid $M$, we denote by $M^{(A)}$ the set of functions $f: A \rightarrow M$ with finite support.

- Let $\alpha \in M^{(A)}$, we denote by $|\alpha|$ the (finite) sum $\sum_{a \in A} \alpha(a)$.

Remark 2.1. In order to avoid cumbersome notation we limit ourself to finitely generated rings and algebras, but we remark that our results remain valid over not finitely generated rings and algebras, unless otherwise stated.

\section{Symmetric FunCtions}

Set $\Lambda_{n}:=\mathbb{K}\left[y_{1}, \ldots, y_{n}\right]^{S_{n}}$ for the ring of symmetric polynomials in $n$ variables: it is freely generated by the elementary symmetric functions $e_{1}, \ldots, e_{n}$ given by the equality

$$
\sum_{k=0}^{n} t^{k} e_{k}:=\prod_{i=1}^{n}\left(1+t y_{i}\right)
$$

Here $e_{0}=1$ and $t$ is an independent variable (see [7). Furthermore one has

$$
e_{k}\left(y_{1}, \ldots, y_{n}\right)=\sum_{i_{1}<i_{2}<\cdots<i_{k} \leq n} y_{i_{1}} y_{i_{2}} \cdots y_{i_{k}}
$$

The action of $S_{n}$ on $\mathbb{K}\left[y_{1}, y_{2}, \ldots, y_{n}\right]$ preserves the usual degree. We denote by $\Lambda_{n}^{k}$ the $\mathbb{K}$-submodule of invariants of degree $k$. 
Let $q_{n}: \mathbb{K}\left[y_{1}, y_{2}, \ldots, y_{n}\right] \rightarrow \mathbb{K}\left[y_{1}, y_{2}, \ldots, y_{n-1}\right]$ be given by

$$
\left\{\begin{array}{l}
y_{n} \mapsto 0 \\
y_{i} \mapsto y_{i} \text { for } i=1, \ldots, n-1
\end{array}\right.
$$

for $i=1, \ldots, n-1$. One has $q_{n}\left(\Lambda_{n}^{k}\right)=\Lambda_{n-1}^{k}$ and it is easy to see that $\Lambda_{n}^{k} \cong \Lambda_{k}^{k}$ for all $n \geq k$. Denote by $\Lambda^{k}$ the limit of the inverse system obtained in this way.

Definition 3.1. The ring $\Lambda:=\bigoplus_{k \geq 0} \Lambda^{k}$ is called the ring of symmetric functions (over $\mathbb{K}$ )

It can be shown 7 that $\Lambda_{R}$ is a polynomial ring, freely generated by the (limits of the) $e_{k}$, that are given by

$$
\sum_{k=0}^{\infty} t^{k} e_{k}:=\prod_{i=1}^{\infty}\left(1+t x_{i}\right)
$$

Furthermore the kernel of the projection $\pi_{n}: \Lambda \rightarrow \Lambda_{n}$ is generated by the $e_{n+k}$, where $k \geq 1$.

We have another distinguished kind of functions in $\Lambda_{n}$ beside the elementary symmetric ones: the power sums, for any $r \in \mathbb{N}$ the $r$-th power sum is

$$
p_{r}:=\sum_{i \geq 1} y_{i}^{r}
$$

Let $g \in \Lambda_{n}$, set $g \cdot p_{r}=g\left(y_{1}^{r}, y_{2}^{r}, \ldots, y_{n}^{r}\right)$, this is again a symmetric function. Since the $e_{i}$ generate $\Lambda_{n}$ we have that $g \cdot p_{r}$ can be expressed as a polynomial in the $e_{i}$ we set

$$
P_{h, k}:=e_{h} \cdot p_{k}
$$

to denote it.

The monomials form a $\mathbb{K}$-basis of $\mathbb{K}\left[y_{1}, \ldots, y_{n}\right]$ permuted by $S_{n}$. Thus, the sums of monomials over the orbits form a $\mathbb{K}$-basis of the ring $\Lambda_{n}$ and their limits form a basis of $\Lambda$. Let $y_{1}^{\lambda_{1}} y_{2}^{\lambda_{2}} \cdots y_{n}^{\lambda_{n}}$ be a monomial, after a suitable permutation we can suppose $\lambda_{1} \geq \lambda_{2} \geq \cdots \geq \lambda_{n} \geq 0$. We set $m_{\lambda}$ for the orbit sum corresponding to such $\lambda=\left(\lambda_{1}, \lambda_{2}, \ldots, \lambda_{n}\right) \in \mathbb{N}^{n}$ then

$$
\mathcal{P}_{n}=\left\{m_{\lambda}: \lambda_{1} \geq \lambda_{2} \geq \cdots \geq \lambda_{n} \geq 0, \lambda_{i} \in \mathbb{N}\right\}
$$

and

$$
\mathcal{P}_{n, k}=\left\{m_{\lambda}: \sum_{i} \lambda_{i}=k\right\}
$$

are $\mathbb{K}$-bases of $\Lambda_{n}$ and $\Lambda_{n}^{k}$ respectively. As before the limits of the $m_{\lambda}$ form a basis of $\Lambda$ and $\Lambda^{k}$ and $\operatorname{ker}\left(\Lambda \rightarrow \Lambda_{n}\right)$ has basis $\left\{m_{\lambda}: \exists k>n\right.$ with $\left.\lambda_{k}>0\right\}$

\section{Symmetric Tensors on Free Algebras}

We set here a generalization of $\Lambda$ and $\Lambda_{n}$, our exposition will be streamlined on the one given in the previous section.

Let $F:=\mathbb{K}\left\{x_{1}, \ldots, x_{m}\right\}$ be a free associative non commutative algebra. Let $k \in$ $\mathbb{N}$, we denote by $\mathbf{f}$ the sequence $\left(f_{1} \ldots, f_{k}\right)$ in $F$ and by $\alpha$ the element $\left(\alpha_{1}, \ldots, \alpha_{k}\right) \in$ 
$\mathbb{N}^{k}$, with $|\alpha|:=\sum \alpha_{j} \leq n$. Let $t_{1}, \ldots, t_{k}$ be commuting independent variables, we set as usual $t^{\alpha}:=\prod_{i} t_{i}^{\alpha_{i}}$. We define elements $e_{\alpha}^{n}(\mathbf{f}) \in T S_{\mathbb{K}}^{n}(F)$ by

$$
\sum_{\alpha} t^{\alpha} \otimes e_{\alpha}^{n}(\mathbf{f}):=\left(1+\sum_{h} t_{h} \otimes f_{h}\right)^{\otimes n}
$$

Example 4.1. Let $f, g \in F$ then

$$
\begin{aligned}
e_{(2,1)}^{3}(f, g) & =f \otimes f \otimes g+f \otimes g \otimes f+g \otimes f \otimes f \\
e_{(2,1)}^{4}(f, g) & =f \otimes f \otimes g \otimes 1+f \otimes g \otimes f \otimes 1+g \otimes f \otimes f \otimes 1 \\
& +f \otimes f \otimes 1 \otimes g+f \otimes g \otimes 1 \otimes f+g \otimes f \otimes 1 \otimes f \\
& +f \otimes 1 \otimes f \otimes g+f \otimes 1 \otimes g \otimes f+g \otimes 1 \otimes f \otimes f \\
& +1 \otimes f \otimes f \otimes g+1 \otimes f \otimes g \otimes f+1 \otimes g \otimes f \otimes f
\end{aligned}
$$

Lemma 4.2. The element $e_{\left(\alpha_{1}, \ldots, \alpha_{k}\right)}^{n}\left(f_{1}, \ldots, f_{k}\right)$ is the orbit sum (under the considered action of $S_{n}$ ) of

$$
f_{1}^{\otimes \alpha_{1}} \otimes f_{2}^{\otimes \alpha_{2}} \cdots \otimes f_{k}^{\otimes \alpha_{k}} \otimes 1^{\otimes\left(n-\sum_{i} \alpha_{i}\right)}
$$

Proof. Let $E$ be the set of mappings $\phi:\{1, \ldots, n\} \rightarrow\{1, \ldots, k+1\}$. We define a mapping $\phi \mapsto \phi^{*}$ of $E$ into $\mathbb{N}^{k+1}$ by putting $\phi^{*}(i)$ equal to the cardinality of $\phi^{-1}(i)$. For two elements $\phi_{1}, \phi_{2}$ of $E$, to satisfy $\phi_{1}^{*}=\phi_{2}^{*}$ it is necessary and sufficient that there should exist $\sigma \in S_{n}$ such that $\phi_{2}=\phi_{1} \circ \sigma$. Set $f_{k+1}:=1_{R}$ and $E(\alpha):=\left\{\phi \in E \mid \phi^{*}=\left(\alpha_{1}, \ldots, \alpha_{k}, n-\sum_{i} \alpha_{i}\right)\right\}$, then we have

$$
e_{\alpha}(\mathbf{f})=\sum_{\phi \in E(\alpha)} f_{\phi(1)} \otimes f_{\phi(2)} \otimes \cdots \otimes f_{\phi(n)}
$$

and the lemma is proved.

Definition 4.3. Let $\Upsilon$ denote the set of monomials in $F$. For $v \in \Upsilon$ let $\partial_{i}(v)$ denote the degree of $v$ in $x_{i}$, for all $i=1, \ldots, m$. We set

$$
\partial(v):=\left(\partial_{1}(v), \ldots, \partial_{m}(v)\right)
$$

for its multidegree. The total degree of $v$ is $\sum_{i} \partial_{i}(v)$. We denote by $\Upsilon^{+}$be the set of monomials of positive degree.

Is is clear that $\Upsilon$ is a $\mathbb{K}$-basis of $F$ so that $\Upsilon_{n}:=\left\{v_{1} \otimes v_{2} \otimes \cdots \otimes v_{n}: v_{j} \in \Upsilon\right\}$ is a $\mathbb{K}$-basis of $T_{\mathbb{K}}^{n}(F)$ permuted by $S_{n}$. Thus, the sums of the elements of $\Upsilon_{n}$ over their orbits form a $\mathbb{K}$-basis of the symmetric tensors $T S_{\mathbb{K}}^{n}(F)$.

Let $\alpha \in \mathbb{N}^{\left(\Upsilon^{+}\right)}$, then there exist $k \in \mathbb{N}$ and $v_{1}, \ldots, v_{k} \in \Upsilon^{+}$such that $\alpha\left(v_{i}\right)=$ $\alpha_{i} \neq 0$ for $i=1, \ldots, k$ and $\alpha(\Upsilon)=0$ when $v \neq v_{1}, \ldots, v_{k}$. We set

$$
e_{\alpha}^{n}:=e_{\left(\alpha_{1}, \ldots, \alpha_{k}\right)}^{n}\left(v_{1}, \ldots, v_{k}\right) \text {, }
$$

then

$$
\sum_{|\alpha| \leq n} t^{\alpha} \otimes e_{\alpha}^{n}=\left(1+\sum_{v \in \Upsilon^{+}} t_{v} \otimes v\right)^{\otimes n}
$$

where $t_{v}$ are commuting independent variables indexed by monomials and

$$
t^{\alpha}:=\prod_{v \in \Upsilon^{+}} t_{v}^{\alpha(v)}
$$


for all $\alpha \in \mathbb{N}^{\left(\Upsilon^{+}\right)}$.

Proposition 4.4. The set

$$
\mathcal{B}_{n}:=\left\{e_{\alpha}^{n}:|\alpha| \leq n\right\}
$$

is a $\mathbb{K}$-basis of $T S_{\mathbb{K}}^{n}(F)$.

Proof. By Lemma 4 and (4.3), the $e_{\alpha}^{n}$ are a complete system of representatives (for the action of $S_{n}$ ) of the orbit sums of the elements of $\Upsilon_{n}$.

Definition 4.5. We define a $S_{n}$-invariant multidegree on $T^{n}(F)$ by

$$
\partial\left(1^{\otimes i} \otimes x_{j} \otimes 1^{n-i-1}\right):=\partial\left(x_{j}\right)
$$

for all $i, j$. We denote by $T_{\mathbb{K}}^{n}(F)_{\delta}\left(\operatorname{resp} . T S_{\mathbb{K}}^{n}(F)_{\delta}\right)$ the linear span of the elements of multidegree $\delta \in \mathbb{N}^{m}$.

Proposition 4.6. The set

$$
\mathcal{B}_{n, \delta}:=\left\{e_{\alpha}^{n}:|\alpha| \leq n \text { and } \partial\left(e_{\alpha}\right)=\delta\right\}
$$

is a $\mathbb{K}$-basis of $T S_{\mathbb{K}}^{n}(F)_{\delta}$ for all $\delta \in \mathbb{N}^{m}$.

Proof. Observe that $\partial\left(e_{\alpha}\right)=\sum_{v \in \Upsilon+} \alpha_{v} \partial(v)$ and apply the above Proposition.

\section{Generators}

First of all we compute the product between the elements of $\mathcal{B}_{n}$

Proposition 5.1 (Product Formula). Let $h, k \in \mathbb{N}, \alpha \in \mathbb{N}^{h}, \beta \in \mathbb{N}^{k}$ be such that $|\alpha|,|\beta| \leq n$. Let $r_{1}, \ldots, r_{h}, s_{1}, \ldots, s_{k} \in F$. Set again

$$
e_{\alpha}^{n}(\mathbf{r}):=e_{\left(\alpha_{1}, \ldots, \alpha_{h}\right)}^{n}\left(r_{1}, \ldots, r_{h}\right) \text { and } e_{\beta}^{n}(\mathbf{s}):=e_{\left(\beta_{1}, \ldots, \beta_{k}\right)}^{n}\left(s_{1}, \ldots, s_{k}\right)
$$

then

$$
e_{\alpha}^{n}(\mathbf{r}) e_{\beta}^{n}(\mathbf{s})=\sum_{\gamma} e_{\gamma}(\mathbf{r}, \mathbf{s}, \mathbf{r s})
$$

where

$$
\begin{aligned}
\text { rs } & :=\left(r_{1} s_{1}, r_{1} s_{2}, \ldots, r_{1} s_{k}, r_{2} s_{1}, \ldots, r_{2} s_{k}, \ldots, r_{h} s_{k}\right) \\
\gamma & :=\left(\gamma_{10}, \ldots, \gamma_{h 0}, \gamma_{01}, \ldots, \gamma_{0 k}, \gamma_{11}, \gamma_{12}, \ldots, \gamma_{h k}\right)
\end{aligned}
$$

are such that

$$
\left\{\begin{array}{l}
\gamma_{i j} \in \mathbb{N} \\
|\gamma| \leq n \\
\sum_{j=0}^{k} \gamma_{i j}=\alpha_{i} \text { for } i=1, \ldots, h \\
\sum_{i=0}^{h} \gamma_{i j}=\beta_{j} \text { for } j=1, \ldots, k .
\end{array}\right.
$$

Proof. Let $t_{1}, t_{2}$ be two commuting independent variables and let $a, b \in F$ then one has

$$
\left(1+t_{1} \otimes a\right)^{\otimes n}\left(1+t_{2} \otimes b\right)^{\otimes n}=\left(1+t_{1} \otimes a+t_{2} \otimes b+t_{1} t_{2} \otimes a b\right)^{\otimes n}
$$


hence

$$
\begin{aligned}
\left(1+\sum_{i=1}^{n} t_{1}^{i} \otimes e_{i}^{n}(a)\right)\left(1+\sum_{j=1}^{n} t_{2}^{j}\right. & \left.\otimes e_{j}^{n}(b)\right) \\
& =1+\sum_{i, j} t_{1}^{i} t_{2}^{j} \otimes e_{i}^{n}(a) e_{j}^{n}(b) \\
& =1+\sum_{l_{1}, l_{2}, l_{12}} t_{1}^{l_{1}+l_{12}} t_{2}^{l_{2}+l_{12}} \otimes e_{\left(l_{1}, l_{2}, l_{12}\right)}^{n}(a, b, a b)
\end{aligned}
$$

The desired equation the easily follows.

Remark 5.2. The above Product Formula can be derived from the one of N.Roby 13] in the context of divided powers. It has been also derived by Ziplies in his papers [18] on the divided powers algebra $\widehat{\Gamma}$.

Corollary 5.3. Let $k \in \mathbb{N}, a_{1}, \ldots, a_{k} \in F, \alpha=\left(\alpha_{1}, \ldots, \alpha_{k}\right) \in \mathbb{N}^{k}$ with $|\alpha| \leq n$. Then $e_{\left(\alpha_{1}, \ldots, \alpha_{k}\right)}^{n}\left(a_{1}, \ldots, a_{k}\right)$ belongs to the subalgebra of $T S_{\mathbb{K}}^{n}(F)$ generated by the $e_{i}^{n}(v)$, where $i=1, \ldots, n$ and $v$ is a monomial in the $a_{1}, \ldots, a_{k}$.

Proof. We prove the claim by induction on $|\alpha|$ assuming that $\alpha_{i}>0$ for all $i$. (note that $1 \leq k \leq \sum_{j} \alpha_{j}$ ). Since $n$ is fixed we suppress the superscript $n$ for all the proof.

If $\sum_{j} \alpha_{j}=1$ then $k=1$ and $e_{\left(\alpha_{1}, \ldots, \alpha_{k}\right)}\left(a_{1}, \ldots, a_{k}\right)=e_{1}\left(a_{1}\right)$. Suppose the claim true for all $e_{\left(\beta_{1}, \ldots, \beta_{h}\right)}\left(b_{1}, \ldots, b_{h}\right)$ with $b_{1}, \ldots, b_{h} \in F$ and $|\beta|<|\alpha|$. Let $k, a_{1}, \ldots, a_{k}, \alpha$ be as in the statement, then we have by the Product Formula

$$
\begin{gathered}
e_{\alpha_{1}}\left(a_{1}\right) e_{\left(\alpha_{2}, \ldots, \alpha_{k}\right)}\left(a_{2}, \ldots, a_{k}\right)= \\
=e_{\left(\alpha_{1}, \ldots, \alpha_{k}\right)}\left(a_{1}, \ldots, a_{k}\right)+\sum e_{\gamma}\left(a_{1}, \ldots, a_{k}, a_{1} a_{2}, \ldots, a_{1} a_{k}\right),
\end{gathered}
$$

where

$$
\gamma=\left(\gamma_{10}, \gamma_{01}, \ldots, \gamma_{0 h}, \gamma_{11}, \gamma_{12}, \ldots, \gamma_{1 h}\right)
$$

with $h=k-1, \sum_{j=0}^{h} \gamma_{1 j}=\alpha_{1}$ with $\sum_{j=1}^{h} \gamma_{1 j}>0$, and $\gamma_{0 j}+\gamma_{1 j}=\alpha_{j}$ for $j=1, \ldots, h$. Thus

$$
\gamma_{10}+\gamma_{01}+\cdots+\gamma_{0 h}+\gamma_{11}+\cdots+\gamma_{1 h}=\sum_{j} \alpha_{j}-\sum_{j=1}^{h} \gamma_{1 j}<\sum_{j} \alpha_{j}
$$

Hence

$$
\begin{gathered}
e_{\left(\alpha_{1}, \ldots, \alpha_{k}\right)}\left(a_{1}, \ldots, a_{k}\right)= \\
e_{\alpha_{1}}\left(a_{1}\right) e_{\left(\alpha_{2}, \ldots, \alpha_{k}\right)}\left(a_{2}, \ldots, a_{k}\right)-\sum e_{\gamma}\left(a_{1}, \ldots, a_{k}, a_{1} a_{2}, a_{1} a_{3}, \ldots, a_{1} a_{k}\right),
\end{gathered}
$$

where $|\gamma|=\sum_{r, s} \gamma_{r s}<|\alpha|$. So the claim follows by induction hypothesis.

Corollary 5.4. The algebra of symmetric tensors $T S^{n}(F)$ of order $n$ is generated by the $e_{i}^{n}(v)$ where $1 \leq i \leq n$ and $v \in \Upsilon^{+}$

Proof. It follows from the above corollary applied to the elements of the basis $\mathcal{B}_{n}$

Remark 5.5. The above corollaries can be proved using Cor(4.1) and Cor(4.5) in 18 
Lemma 5.6. For all $f \in F$, and $k, h \in \mathbb{N}, e_{h}^{n}\left(f^{k}\right)$ belongs to the subalgebra of $T S_{\mathbb{K}}^{n}(F)$ generated by the $e_{j}^{n}(f)$.

Proof. Let $f \in F$ there is an obvious $S_{n}$-equivariant morphism

$$
\rho_{f}:\left\{\begin{array}{l}
\mathbb{K}\left[y_{1}, \ldots, y_{m}\right] \rightarrow T_{\mathbb{K}}^{n} F, \\
y_{h} \mapsto 1^{\otimes(h-1)} \otimes f \otimes 1^{\otimes(n-h)}
\end{array}\right.
$$

induced by the evaluation $\mathbb{K}[x] \rightarrow \mathbb{K}[f]$. It is the clear that $\rho_{f}\left(e_{i}\right)=e_{i}^{n}(f)$ so that

$$
e_{h}^{n}\left(f^{k}\right)=\rho_{f}\left(e_{h} \cdot p_{k}\right)=\rho_{f}\left(P_{h, k}\right)
$$

and the result is proved.

Definition 5.7. A monomial $v \in \Upsilon^{+}$is called primitive if it is not the power of another one.

Example 5.8. $x_{1} x_{2} x_{1} x_{2}$ is not primitive while $x_{1} x_{2} x_{1} x_{1}$ is primitive.

We have then the following refinement of Cor 5.4

Theorem 5.9 (Generators). The algebra $T S_{\mathbb{K}}^{n}(F)$ is generated by $e_{i}^{n}(v)$ with $1 \leq$ $i \leq n$ and $v$ primitive

Proof. It follows from Cor 5.4 and Lemma 5.6

5.1. Abelianization. Given any $\mathbb{K}$-algebra $R$ one can form the bilateral ideal $[R, R]$ generated by the commutators $[a, b]:=a b-b a, a, b \in R$.

We set $R^{a b}:=R /[R, R]$ and call it the abelianization of $R$. The abelianization of $R$ is commutative and together with the homomorphism $\mathfrak{a} \mathfrak{b}: R \longrightarrow R /[R, R]$ has the universal property that the map $\mathcal{C}_{\mathbb{K}}\left(R^{a b}, S\right) \rightarrow \mathcal{N}_{\mathbb{K}}(R, S)$ given by $\varphi \mapsto \varphi \circ \mathfrak{a b}$ is an isomorphism for all $S \in \mathcal{C}_{\mathbb{K}}$.

Definition 5.10. Consider $\Upsilon / \sim$ the set of the equivalence classes of monomials $v \in \Upsilon^{+}$where $v \sim v^{\prime}$ if and only if there a cyclic permutation $\sigma$ such that $\sigma(v)=v^{\prime}$. We set $\Psi$ to denote the set of equivalence classes in $\Upsilon^{+} / \sim$ made of primitive monomials

We are now able to give the main result of this paragraph.

Theorem 5.11. The algebra $\left(T S_{\mathbb{K}}^{n}(F)\right)^{a b}$ is generated by $e_{i}^{n}(v)$ with $1 \leq i \leq n$ and $v$ that varies in a complete set of representatives of $\Psi$.

Proof. Let $\mathfrak{a} \mathfrak{b}: T S_{\mathbb{K}}(F) \longrightarrow\left(T S_{\mathbb{K}}^{n}(F)\right)^{a b}$ be the canonical projection, using (5.2) it easy to see that

$$
\mathfrak{a} \mathfrak{b}\left(e_{i}^{n}(r s)\right)=\mathfrak{a} \mathfrak{b}\left(e_{i}^{n}(s r)\right)
$$

for all $1 \leq i \leq n$ and $r, s \in F$. The result then follows from Th 5.9 and Prop.?? by the surjectivity of $\mathfrak{a} \mathfrak{b}$.

\section{Polynomial Laws}

To link symmetric tensors to linear representations we shall use the determinant so that we are lead to the topic of polynomial laws: we recall the definition of this kind of map between $\mathbb{K}$-modules that generalizes the usual polynomial mapping between free $\mathbb{K}$-modules (see [2, 12, 13]). 
Definition 6.1. Let $A$ and $B$ be two $\mathbb{K}$-modules. A polynomial law $\varphi$ from $A$ to $B$ is a family of mappings $\varphi_{L}: L \otimes_{\mathbb{K}} A \longrightarrow L \otimes_{\mathbb{K}} B$, with $L \in \mathcal{C}_{\mathbb{K}}$ such that the following diagram commutes

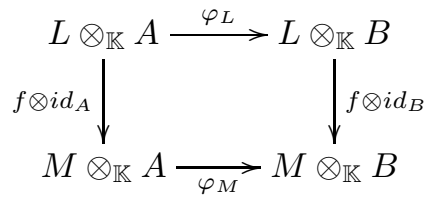

for all $L, M \in \mathcal{C}(\mathbb{K})$ and all $f \in \mathcal{C}_{\mathbb{K}}(L, M)$.

Definition 6.2. Let $n \in \mathbb{N}$, if $\varphi_{L}(a u)=a^{n} \varphi_{L}(u)$, for all $a \in L, u \in L \otimes_{\mathbb{K}} A$ and all $L \in \mathcal{C}_{\mathbb{K}}$, then $\varphi$ will be said homogeneous of degree $n$.

Definition 6.3. If $A$ and $B$ are two $\mathbb{K}$-algebras and

$$
\begin{cases}\varphi_{L}(x y) & =\varphi_{L}(x) \varphi_{L}(y) \\ \varphi_{L}\left(1_{L \otimes A}\right) & =1_{L \otimes B}\end{cases}
$$

for $L \in \mathcal{C}_{\mathbb{K}}$ and for all $x, y \in L \otimes_{\mathbb{K}} A$, then $\varphi$ is called multiplicative.

Remark 6.4. A polynomial law $\varphi: A \rightarrow B$ is a natural transformation $-\otimes_{\mathbb{K}} A \rightarrow$ $-\otimes_{\mathbb{K}} B$.

Let $A$ and $B$ be two $\mathbb{K}$-modules and $\varphi: A \rightarrow B$ be a polynomial law. The following result on polynomial laws is a restatement of Théorème I.1 of [12].

Theorem 6.5. Let $S$ be a set.

(1) Let $L=\mathbb{K}\left[x_{s}\right]_{s \in S}$ and let $\left\{a_{s}: s \in S\right\} \subset A$ be such that $a_{s}=0$ except for a finite number of $s \in S$, then there exist $\varphi_{\xi}\left(\left(a_{s}\right)\right) \in B$, with $\xi \in \mathbb{N}^{(S)}$, such that:

$$
\varphi_{L}\left(\sum_{s \in S} x_{s} \otimes a_{s}\right)=\sum_{\xi \in \mathbb{N}^{(S)}} x^{\xi} \otimes \varphi_{\xi}\left(\left(a_{s}\right)\right),
$$

where $x^{\xi}:=\prod_{s \in S} x_{s}^{\xi_{s}}$.

(2) Let $R$ be any commutative $\mathbb{K}$-algebra and let $\left(r_{s}\right)_{s \in S} \subset R$, then:

$$
\varphi_{R}\left(\sum_{s \in S} r_{s} \otimes a_{s}\right)=\sum_{\xi \in \mathbb{N}(S)} r^{\xi} \otimes \varphi_{\xi}\left(\left(a_{s}\right)\right),
$$

where $r^{\xi}:=\prod_{s \in S} r_{s}^{\xi_{s}}$

(3) If $\varphi$ is homogeneous of degree $n$, then one has $\varphi_{\xi}\left(\left(a_{s}\right)\right)=0$ if $|\xi|$ is different from $n$. That is:

$$
\varphi_{R}\left(\sum_{a \in A} r_{a} \otimes a\right)=\sum_{\xi \in \mathbb{N}^{(A)},|\xi|=n} r^{\xi} \otimes \varphi_{\xi}((a)) .
$$

In particular, if $\varphi$ is homogeneous of degree 0 or 1 , then it is constant or linear, respectively.

Remark 6.6. The above theorem means that a polynomial law $\varphi: A \rightarrow B$ is completely determined by its coefficients $\varphi_{\xi}\left(\left(a_{s}\right)\right)$, with $\left(a_{s}\right)_{s \in S} \in S^{(\mathbb{N})}$. 
Remark 6.7. If $A$ is a free $\mathbb{K}$-module and $\left\{a_{t}: t \in T\right\}$ is a basis of $A$, then $\varphi$ is completely determined by its coefficients $\varphi_{\xi}\left(\left(a_{t}\right)\right)$, with $\xi \in \mathbb{N}^{(T)}$. If also $B$ is a free $\mathbb{K}$-module with basis $\left\{b_{u}: u \in U\right\}$, then $\varphi_{\xi}\left(\left(a_{t}\right)\right)=\sum_{u \in U} \lambda_{u}(\xi) b_{u}$. Let $a=\sum_{t \in T} \mu_{t} a_{t} \in A$. Since only a finite number of $\mu_{t}$ and $\lambda_{u}(\xi)$ are different from zero, the following makes sense:

$$
\begin{aligned}
\varphi(a)=\varphi\left(\sum_{t \in T} \mu_{t} a_{t}\right)=\sum_{\xi \in \mathbb{N}^{(T)}} \mu^{\xi} \varphi_{\xi}\left(\left(a_{t}\right)\right) & =\sum_{\xi \in \mathbb{N}^{(T)}} \mu^{\xi}\left(\sum_{u \in U} \lambda_{u}(\xi) b_{u}\right) \\
& =\sum_{u \in U}\left(\sum_{\xi \in \mathbb{N}^{(T)}} \lambda_{u}(\xi) \mu^{\xi}\right) b_{u} .
\end{aligned}
$$

Hence, if both $A$ and $B$ are free $\mathbb{K}$-modules, a polynomial law $\varphi: A \rightarrow B$ is simply a polynomial map.

Definition 6.8. Let $\mathbb{K}$ be a commutative ring.

(1) For $M, N$ two $\mathbb{K}$-modules we set $P^{n}(M, N)$ for the set of homogeneous polynomial laws $M \rightarrow N$ of degree $n$

(2) If $M, N$ are two $\mathbb{K}$-algebras we set $M P^{n}(M, N)$ for the multiplicative homogeneous polynomial laws $M \rightarrow N$ of degree $n$.

The assignment $N \longrightarrow P^{n}(M, N)$ (resp. $\left.N \longrightarrow M P^{n}(M, N)\right)$ determines a functor from $\mathbb{K}$-modules (resp. $\mathbb{K}$-algebras) to Set.

\section{Divided Powers}

The functors just introduced in Def 6.8 are represented by the divided powers which we introduce right now in the stream.

Definition 7.1. Let $\mathbb{K}$ be any commutative ring with identity. For a $\mathbb{K}$-module $M$ let $\Gamma_{\mathbb{K}}(M)$ denote its divided powers algebra (see [2, 12] and [13]). $\Gamma_{\mathbb{K}}(M)$ is an associative and commutative $\mathbb{K}$-algebra with identity $1_{\mathbb{K}}$ and product $\times$, with generators $m^{(k)}$, with $m \in M, k \in \mathbb{Z}$ and relations, for all $m, n \in M$ :

(1) $m^{(i)}=0, \forall i<0$;

(2) $m^{(0)}=1_{\mathbb{K}}, \forall m \in M$;

(3) $(\alpha m)^{(i)}=\alpha^{i} m^{(i)}, \forall \alpha \in \mathbb{K}, \forall i \in \mathbb{N}$;

(4) $(m+n)^{(k)}=\sum_{i+j=k} m^{(i)} n^{(j)}, \forall k \in \mathbb{N}$;

(5) $m^{(i)} \times m^{(j)}=\left(\begin{array}{c}i+j \\ i\end{array}\right) m^{(i+j)}, \forall i, j \in \mathbb{N}$.

The $\mathbb{K}$-module $\Gamma_{\mathbb{K}}(M)$ is generated by finite products $\times_{i \in I} x_{i}^{\left(\alpha_{i}\right)}$ of the above generators. The divided powers algebra $\Gamma_{\mathbb{K}}(M)$ is a $\mathbb{N}$-graded algebra with homogeneous components $\Gamma_{\mathbb{K}}^{k}(M),(k \in \mathbb{N})$, the submodule generated by $\left\{\times_{i \in I} x_{i}^{\left(\alpha_{i}\right)}:|\alpha|=\right.$ $\left.\sum_{i} \alpha_{i}=k\right\}$. One easily check that $\Gamma_{\mathbb{K}}$ is a functor from $\mathbb{K}$-modules to commutative graded $\mathbb{K}$-algebras.

7.1. Universal properties. The following properties give the motivation for the introduction of divided powers in our setting.

7.1.1. $\quad \Gamma_{\mathbb{K}}^{n}$ is a covariant functor from $\operatorname{Mod}_{\mathbb{K}}$ to $\operatorname{Mod}_{\mathbb{K}}$ and one can easily check that it preserves surjections. The map $\gamma^{n}: r \mapsto r^{(n)}$ is a polynomial law $M \rightarrow \Gamma_{\mathbb{K}}^{n}(M)$ homogeneous of degree $n$ : we call it the universal one. We have an isomorphisms $\operatorname{Mod}_{\mathbb{K}}\left(\Gamma_{\mathbb{K}}^{n}(M), N\right) \rightarrow P_{\mathbb{K}}^{n}(M, N)$ given by $\phi \mapsto \phi \circ \gamma^{n}$. 
7.1.2. If $R$ is $\mathbb{K}$-algebra then $\Gamma_{\mathbb{K}}^{n}(R)$ inherits a structure of $\mathbb{K}$-algebra by induced by setting $a^{(n)} b^{(n)}=(a b)^{(n)}$. The unit in $\Gamma_{\mathbb{K}}^{n}(R)$ is $1^{(n)}$. It was proven by N.Roby 13. that in this way $R \rightarrow \Gamma_{\mathbb{K}}^{n}(R)$ gives a functor from $\mathbb{K}$-algebras to $\mathbb{K}$-algebras such that $\gamma^{n}(a) \gamma^{n}(b)=\gamma^{n}(a b), \forall a, b \in R$. Hence $\gamma^{n} \in M P_{\mathbb{K}}^{n}\left(M, \Gamma_{\mathbb{K}}^{n}(M)\right)$ and the $\operatorname{map} \mathcal{N}_{\mathbb{K}}\left(\Gamma_{\mathbb{K}}^{n}(R), S\right) \rightarrow M P_{\mathbb{K}}(R, S)$ given by $\phi \mapsto \phi \circ \gamma^{n}$ is an isomorphism for all $R, S \in \mathcal{N}_{\mathbb{K}}$.

7.2. Flatness and Symmetric Tensors. Let $M \in M o d_{\mathbb{K}}$. Consider again the symmetric tensors $T S_{\mathbb{K}}^{n}(M)$ of order $n$. When $M \in \mathcal{N}_{\mathbb{K}}$ (resp. $M \in \mathcal{C}_{\mathbb{K}}$ ) then $T S_{\mathbb{K}}^{n}(M) \in \mathcal{N}_{\mathbb{K}}\left(\right.$ resp. $T S_{\mathbb{K}}^{n}(M) \in \mathcal{C}_{\mathbb{K}}$ ). The homogeneous polynomial law $M \rightarrow$ $T S_{\mathbb{K}}^{n}(M)$ given by $x \mapsto x^{\otimes n}$ gives a morphism $\tau_{n}: \Gamma_{\mathbb{K}}^{n}(M) \rightarrow T S_{\mathbb{K}}^{n}(M)$ that is an isomorphism when $M$ is flat over $\mathbb{K}$.(see [4 5.5.2.5 pag.123). Note that $\tau_{n}\left(r^{(n)}\right)=$ $r^{\otimes n}$ (see [2]) and observe that this implies that $\tau_{n}(1+t \otimes r)^{(n)}=(1+t \otimes r)^{\otimes n}$ where $t$ is a commuting independent variable so that

$$
\tau_{n}\left(1^{(n-j)} r^{(j)}\right)=e_{i}^{n}(r)
$$

for all $\mathbb{K}$-algebra $R, \forall r \in R$ and $j=1, \ldots, n$.

Corollary 7.2. Let $R$ be a $\mathbb{K}$-algebra generated by $\left\{r_{i}\right\}_{i \in I}$ then

(1) $\Gamma_{\mathbb{K}}^{n}(R)$ is generated by $1^{(n-j)} v^{(j)}$ where $1 \leq j \leq n$ and $v$ varies in the set of primitive monomials in $\left\{r_{i}\right\}_{i \in I}$

(2) $\left(\Gamma_{\mathbb{K}}^{n}(R)\right)^{a b}$ is generated by $1^{(n-j)} v^{(j)}$ where $v$ varies in a complete set of representative of equivalence classes (under cyclic permutations) of primitive monomials in the $\left\{r_{i}\right\}_{i \in I}$.

Proof. Given a $\mathbb{K}$-algebra $R$ and a set $\left\{r_{i}\right\}_{i \in I}$ of its generators we have a surjective homomorphism $F_{I}:=\mathbb{K}\left\{x_{\alpha}\right\} \rightarrow R$ hence another one $T S_{\mathbb{K}}^{n}\left(F_{I}\right) \cong \Gamma_{\mathbb{K}}^{n}\left(F_{I}\right) \rightarrow \Gamma_{\mathbb{K}}^{n}(R)$ and the result follows from Cor 5.9 recalling (7.1). The result on the abelianization follows from $\mathrm{Th} 5.11$ since the abelianization functor preserves surjections.

Remark 7.3. Corollary 17.2(1) is a refinement of Cor.(4.5) in [18.

Corollary 7.4. Let $R$ be commutative.

(1) The ring $T S_{\mathbb{K}}^{n}\left(\mathbb{K}\left[y_{1}, \ldots, y_{m}\right]\right) \cong \mathbb{K}\left[x_{11}, x_{12}, \ldots, x_{m 1}, \ldots, x_{m n}\right]^{S_{n}}$ of the multisymmetric functions also known as the ring of the vector invariants of the symmetric group is generated by the $e_{i}^{n}(v)$ with $v=y_{1}^{\alpha_{1}} \cdots y_{m}^{\alpha_{m}}$ such that $\alpha_{1}, \ldots, \alpha_{m}$ are coprime.

(2) Let $R$ be a commutative $\mathbb{K}$-algebra generated by $\left\{r_{i}\right\}_{i \in I}$ then $\Gamma_{\mathbb{K}}^{n}(R)$ is generated by $1^{(n-j)} v^{(j)}$ where $v=\prod_{i} r_{i}^{\alpha_{i}}$ is such that $\sum_{i} \alpha_{i}$ is finite and the $\alpha_{i}$ are coprime.

Proof. It follows from Th 5.11 since $\Gamma^{n}$ preserves surjections. Write $P=K\left[y_{1}, \ldots, y_{m}\right]$, then $P$ is a free $\mathbb{K}$-module hence the surjection $F \rightarrow P$ induces another surjection $T S^{n} F^{a b} \rightarrow T S^{n} P$. Use Th 5.11 .

Remark 7.5. This gives another proof of Th.1 in 15

Remark 7.6. Corollary 17.4 has been improved by D.Rydh [1] which found a minimal generating set for $T S_{\mathbb{K}}^{n}\left(\mathbb{K}\left[y_{1}, \ldots, y_{m}\right]\right)$. 
7.3. Good Characteristics. In the ring $\Lambda$ of the symmetric functions it holds the following well known Newton's Formulas

$$
(-1)^{k} p_{k+1}+\sum_{i=1}^{k}(-1)^{i} p_{i} e_{k+1-i}=(k+1) e_{k+1}
$$

for all $k>0$. It is clear that these equalities hold also in $\Lambda_{n}$ with $e_{i}=0$ for $i>n$.

Proposition 7.7. Let $R$ be generated by $\left\{r_{i}\right\}_{i \in I}$ as a $\mathbb{K}$-algebra. Let $n$ ! be invertible in $\mathbb{K}$ then $\Gamma_{\mathbb{K}}^{n}(R)$ is generated by $1^{(n-1)} v^{(1)}$ where $v$ varies in the monomials in the $r_{i}$. If $R$ is commutative the monomial $v$ can be taken of degree not greater than $n$.

Proof. In this case we have that Newton's formulas made $p_{1}, p_{2}, \ldots, p_{n}$ a generating set for $\Lambda_{n}$ hence $1^{(n-i)} v^{(i)}$ belongs to the subring generated by the $1^{(n-1)}\left(v^{k}\right)^{(1)}$ and this gives the desired result when combined with the results on generating sets given in the previous paragraphs. If $R$ is commutative the result follows because it holds on $\Gamma_{\mathbb{K}}^{n}\left(\mathbb{K}\left[y_{1}, \ldots, y_{m}\right]\right)$ by E.Noether's bound.

\section{8. $\Gamma^{n} \operatorname{vS} T S^{n}$}

We have seen that $T S_{\mathbb{K}}^{n}(F)$ is generated by $e_{i}^{n}(v)$ with $v$ primitive. Let $R$ be generated as $\mathbb{K}$-algebra by $\left\{r_{i}\right\}_{i \in I}$ and let $\pi: F \rightarrow R$ be a surjective homomorphism, then we have that the image of

$$
T S_{\mathbb{K}}^{n}(\pi): T S_{\mathbb{K}}^{n}(F) \rightarrow T S_{\mathbb{K}}^{n}(R)
$$

is the subalgebra of $T S_{\mathbb{K}}^{n}(R)$ generated by the $T S_{\mathbb{K}}^{n}(\pi)\left(e_{i}^{n}(v)\right)$ but this latter are the orbits sums of $\pi(v)^{\otimes i} \otimes 1^{\otimes(n-i)}$. Hence the image of $T S_{\mathbb{K}}^{n}(\pi)$ is the image of the canonical morphism $\Gamma_{\mathbb{K}}^{n}(R) \rightarrow T S_{\mathbb{K}}^{n}(R)$ corresponding to the polynomial law $r \mapsto r^{\otimes n}$. We have proven the following

Proposition 8.1. Let $F$ be free and let $F \rightarrow R$ be onto. Write $\tau: \Gamma_{\mathbb{K}}(R) \rightarrow$ $T S_{\mathbb{K}}^{n}(R)$ for the canonical homomorphism such that $\tau\left(r^{(n)}\right)=r^{\otimes n}$ for all $r \in R$. Then $\tau\left(\Gamma_{\mathbb{K}}^{n}(R)\right)=T S_{\mathbb{K}}^{n}(\pi)\left(T S_{\mathbb{K}}^{n}(F)\right)$.

\section{Relations: THE FIRST SYZYGY}

We have generators we look for relations between them: the first syzygy.

9.1. The free object. When you are looking for syzygies the first step is usually to find some free objects mapping onto your algebra. The basic idea here is to use the basis $\mathcal{B}_{n}$ we found for $T S_{\mathbb{K}}^{n}(F)$ allowing $n \rightarrow \infty$.

Definition 9.1. Let $F=\mathbb{K}\left\{x_{1}, \ldots, x_{m}\right\}$ as usual.

- Set $\mathfrak{F}$ for the free $\mathbb{K}$-module with basis $\mathcal{B}:=\left\{f_{\alpha}: \alpha \in \mathbb{N}^{\left(\Upsilon^{+}\right)}\right\}$.

- We set $1_{\mathfrak{F}}:=f_{\mathbf{0}}$ where $\mathbf{0} \in \mathbb{N}^{\Upsilon^{+}}$is such that $\mathbf{0}(v)=0$ for all $v \in \Upsilon^{+}$.

- For $i \in \mathbb{N}-\{0\}$ and $v \in \Upsilon^{+}$we denote by $f_{i}(v)$ the element of $\mathcal{B}$ corresponding to $\alpha: \Upsilon^{+} \rightarrow \mathbb{N}$ such that $\alpha(v)=i$ and $\alpha(\mu)=0$ otherwise.

- We set $\mathfrak{F}^{n}$ the sub- $\mathbb{K}-$ module generated by those $f_{\alpha}$ with $\sum_{v \in \Upsilon^{+}} \alpha(v)>$ $n$. 
For all $n \geq 1$ there a split exact sequence of $\mathbb{K}$-modules

$$
0 \longrightarrow \mathfrak{F}^{n} \longrightarrow \mathfrak{F} \stackrel{\sigma_{n}}{\longrightarrow} T S_{\mathbb{K}}^{n} F \longrightarrow 0
$$

where

is given by

$$
\sigma_{n}: \mathfrak{F} \rightarrow T S_{\mathbb{K}}^{n} F
$$

$$
\sigma_{n}: \begin{cases}f_{\alpha} \mapsto e_{\alpha}^{n}, & \text { if } \sum_{v \in \Upsilon^{+}} \alpha(v) \leq n \\ f_{\alpha} \mapsto 0, & \text { otherwise }\end{cases}
$$

Using this splitting one can lift the product of $T S_{\mathbb{K}}^{n} F$ to $\mathfrak{F}$ making it into an associative $\mathbb{K}$-algebra with identity $1_{\mathfrak{F}}$. Observe indeed that the Product Formula stabilizes for $n$ big enough because the number of solutions of (5.1) is finite also if one drops out the constrain $\sum \gamma_{i j} \leq n$. Thus one can express $e_{\alpha}^{n} e_{\beta}^{n}=\sum_{\gamma} e_{\gamma}^{n}$ with respect to $\mathcal{B}_{n}$ using the Product Formula with $n \gg \max \left(\sum_{i} \alpha_{i}, \sum \beta_{j}\right)$ and then define $f_{\alpha} f_{\beta}:=\sigma_{n}^{-1}\left(\sum_{\gamma} e_{\gamma}^{n}\right)=\sum_{\gamma} f_{\gamma}$

Proposition 9.2. The $\mathbb{K}$-algebra $\mathfrak{F}$ is generated by $f_{i}(v)$ where $i \geq 1$ and $v \in \Upsilon^{+}$ is primitive.

Proof. By the very proof of Th 5.9 and the definition of $\mathfrak{F}$ it is possible to express any $f_{\alpha} \in \mathcal{B}$ as an element of the subalgebra generated by the $f_{i}(v)$ of the statement.

Corollary 9.3. The $\mathbb{K}$-algebra $\mathfrak{F}^{a b}$ is generated by $\mathfrak{a b}\left(f_{i}(v)\right)$ where $i \geq 1$ and $v$ that varies in a complete set of representatives of $\Psi$.

Proof. By the above prop. and the section on generators.

9.2. Degree. Few words on degree: we have a multidegree $\partial$ on $T S_{\mathbb{K}}^{n}(F)$ and again we can lift it up to $\mathfrak{F}$ using $\sigma_{n}$ namely by

$$
\partial\left(f_{\alpha}\right):=\sum_{v \in \Upsilon^{+}} \alpha(v) \partial(v)
$$

Definition 9.4. For $\alpha \in \mathbb{N}^{m}$ write $\mathfrak{F}_{\alpha}\left(\operatorname{resp} . T S_{\mathbb{K}}^{n}(F)_{\alpha}\right)$ for the $\mathbb{K}$-submodule of $\mathfrak{F}$ (resp. of $T S_{\mathbb{K}}^{n}(F)$ ) of the elements of multidegree $\alpha$. Analogously we define the total degree of an homogeneous element of $f \in \mathfrak{F}$ to be the sum $\sum_{i} \partial_{i}(f)$.

By construction

$$
\mathfrak{F}=\bigoplus_{\alpha} \mathfrak{F}_{\alpha}
$$

and this make $\mathfrak{F}$ a $\mathbb{N}^{m}$-graded $\mathbb{K}$-algebra as well as

$$
T S_{\mathbb{K}}^{n}(F)=\bigoplus_{\alpha} T S_{\mathbb{K}}^{n}(F)_{\alpha}
$$

The same holds for the abelianizations $\mathfrak{F}^{a b}$ and $T S_{\mathbb{K}}^{n}(F)^{a b}$ as can be easily seen.

Proposition 9.5. One has $\mathfrak{F}_{\alpha} \cong T S_{\mathbb{K}}^{n}(F)_{\alpha}$ for all $\alpha \in \mathbb{N}^{m}$ such that $\sum_{i} \alpha_{i} \leq n$ that is $\operatorname{ker} \sigma_{n} \cap \bigoplus_{\alpha, \sum_{i} \alpha_{i} \leq n} \mathfrak{F}_{\alpha}=\{0\}$. In other words $\mathfrak{F}_{\alpha} \cong T S_{\mathbb{K}}^{n}(F)_{\alpha}, \forall n>\sum_{i} \alpha_{i}$.

Proof. By the definition of degree it is clear that $\mathfrak{F}_{\alpha} \cap \mathfrak{F}^{n}=\{0\}$ for all $n>\sum_{i} \alpha_{i}$ the total degree of the elements of $\mathfrak{F}_{\alpha}$ and the result follows. 
Corollary 9.6. Let $\mathfrak{F}_{k}$ (resp. $\left.T S_{\mathbb{K}}^{n}(F)_{k}\right)$ be the linear span of the elements of total degree $k \in \mathbb{N}$ of $\mathfrak{F}$ (resp. of $T S_{\mathbb{K}}^{n}(F)$ ), then $\mathfrak{F}_{k} \cap \mathfrak{F}^{n}=\{0\}$ for all $n>k$ and $\mathfrak{F}_{k} \cong T S_{\mathbb{K}}^{n}(F)_{k}$ for all $n>k$.

Proof. Observe that $\mathfrak{F}_{k}=\bigoplus_{\alpha, \sum_{i} \alpha_{i}=k} \mathfrak{F}_{\alpha}$ and so on. Then apply the above corollary.

Proposition 9.7. Let $\mathfrak{F}$ be endowed with the above defined product then the sequence 9.1) gives an isomorphism of $\mathbb{N}^{m}$-graded $\mathbb{K}$-algebra $T S_{\mathbb{K}}^{n}(F) \cong \mathfrak{F} / \mathfrak{F}^{n}$. The splitting in (9.1) is not an algebra homomorphism.

Proof. By construction $\sigma_{n}$ is a surjective $\mathbb{N}^{m}$-graded $\mathbb{K}$-algebras homomorphism whose kernel is $\mathfrak{F}^{n}$. Looking at Product Formula one can check that $f_{\alpha} f_{\beta}$ does not belong in general to the submodule generated by those $f_{\gamma}$ such that $\gamma \leq$ $\max \left(\sum_{i} \alpha_{i}, \sum \beta_{j}\right)$ hence the sequence is not split in the category of $\mathbb{K}$-algebras.

\section{Main Result}

We would like to show now that $\mathfrak{F}^{a b}$ is a freely generated by the $\mathfrak{a} \mathfrak{b}\left(f_{i}(v)\right)$ where $i \geq 1$ and $v$ that varies in a complete set of representatives of $\Psi$. In order to prove this result we need some machinery coming from representations theory.

Definition 10.1. We write $f_{i, v}$ for $\mathfrak{a} \mathfrak{b}\left(f_{i}(v)\right)$.

10.1. Generic matrices. This subsection is borrowed from Procesi, see [3] for a recent paper and 8 for the original sources.

Let $A_{n}:=\mathbb{K}\left[\xi_{i j, h}\right]$ be a polynomial ring where $i, j=1, \ldots, n$ and $h=1, \ldots, m$, note that $A_{n}$ is isomorphic to the symmetric $\mathbb{K}$-algebra of the dual of $\operatorname{Mat}(n, \mathbb{K})^{m}$.

Let $F$ be again the free associative $\mathbb{K}$-algebra on $m$ generators then

$$
\mathcal{N}_{\mathbb{K}}(F, \operatorname{Mat}(n, S)) \cong \operatorname{Mat}(n, S)^{m} \cong \mathcal{C}_{\mathbb{K}-a l g}\left(A_{n}, S\right)
$$

for any commutative $\mathbb{K}$-algebra $S$. More precisely set $B_{n}:=\operatorname{Mat}\left(n, A_{n}\right)$ and let $\xi_{h} \in B_{n}$ be given by $\left(\xi_{h}\right)_{i j}=\xi_{i j, h}, \forall i, j, h$, these are called the $(n \times n)$ generic matrices (over $\mathbb{K}$ ) and were introduced by Procesi (see [8]). Let $\pi_{n}$ : $F \rightarrow B_{n}$ be the $\mathbb{K}$-algebra given by $x_{h} \mapsto \xi_{h}$. We have then that given any $\left.\rho \in \operatorname{Hom}_{\mathbb{K}-a l g}(F, \operatorname{Mat}(n, S))\right)$, with $S$ a commutative $\mathbb{K}$-algebra, there is a unique $\bar{\rho} \in \operatorname{Hom}_{\mathbb{K}-a l g}\left(A_{n}, S\right)$ given by $\xi_{i j, h} \mapsto\left(\rho\left(\xi_{h}\right)\right)_{i j}$ and it is such that the following diagram commutes

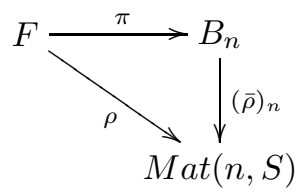

where $(-)_{n}$ denotes the induced map on $n \times n$ matrices. The homomorphism $\pi_{n}$ is called the universal $n$ dimensional representation (for the free algebra). We denote by $\mathcal{G}_{n}$ the subring of $B_{n}$ generated by the generic matrices i.e. the image of $\pi_{n}$. 
10.2. Determinant. The composition $\operatorname{det} \cdot \pi$ gives a multiplicative polynomial law $F \rightarrow A$ homogeneous of degree $n$ hence a unique homomorphism $\delta_{n}: T S_{\mathbb{K}}^{n}(F)^{a b} \rightarrow$ $A$ such that $\delta_{n}\left(\mathfrak{a} \mathfrak{b}\left(e_{n}^{n}(f)\right)\right)=\operatorname{det}\left(\pi_{n}(f)\right)$.

Definition 10.2. Let $C_{n} \subset A_{n}$ be the subalgebra generated by the coefficients of the characteristic polynomials of the elements of $\mathcal{G}_{n}$. We write

$$
\operatorname{det}(t-f)=t^{n}+\sum_{i=1}^{n}(-1)^{i} \varsigma_{i}^{n}(f) t^{n-i}
$$

where $f \in \mathcal{G}_{n}$.

Note that $\delta_{n}\left(\mathfrak{a} \mathfrak{b}\left(e_{i}^{n}(f)\right)\right)=\varsigma_{i}^{n}\left(\pi_{n}(f)\right)$ for all $f \in F$.

By Theorem 5.11 we have that $\delta_{n}\left(T S_{\mathbb{K}}^{n}(F)^{a b}\right)=C_{n}$. If one gives $\xi_{i j, h}$ multidegree $\partial\left(\xi_{i j, h}\right)=\partial\left(x_{h}\right)$ then $A_{n}=\bigoplus_{\alpha \in \mathbb{N}^{m}} A_{n, \alpha}$ is a $\mathbb{N}^{n}$-graded ring with homogeneous components $A_{n, \alpha}$ and one can easily check that $C_{n}=\bigoplus_{\alpha \in \mathbb{N}^{m}} C_{n, \alpha}$ where $C_{n, \alpha}=$ $A_{n, \alpha} \cap C_{n}$ are the homogeneous component.

One checks that $\delta_{n}\left(T S_{\mathbb{K}}^{n}(F)_{\alpha}^{a b}\right)=C_{n, \alpha}$.

For all $n$ there is an homomorphism of $\mathbb{N}^{m}$-graded rings $\varphi_{n}: A_{n} \rightarrow A_{n-1}$ given by $x_{n j, h}, x_{i n, h} \mapsto 0$ and $x_{i j, h} \mapsto x_{i j_{h}}$ for $i, j<n$.

Definition 10.3. We write $C_{\alpha}:=\lim _{n} C_{n, \alpha}$ and $C=\bigoplus_{\alpha \in \mathbb{N}^{m}} C_{\alpha}$ and $\varsigma_{i}(f)=$ $\lim _{n} \varsigma_{i}^{n}(f)$.

Remark 10.4. The algebra $C$ is the graded inverse limit of the $C_{n}$.

Proposition 10.5. The ring $C$ is a polynomial ring freely generated by the $\varsigma_{i, v}$ with $i \geq 1$ and $v$ that varies in a complete set of representatives of $\Psi$.

Proof. By [6], $\S 3,(10)$ and remark loc.cit. and Complements [5] .

10.3. Freeness. We are now in the following situation: there are surjective homomorphisms

$$
\mathfrak{F}^{a b} \stackrel{\tilde{\sigma_{n}}}{\longrightarrow} T S_{\mathbb{K}}^{n}(F)^{a b} \stackrel{\delta_{n}}{\longrightarrow} C_{n}
$$

of $\mathbb{N}^{m}$-graded algebras that commutes with $\varphi_{n}$ hence there is an homomorphism $\delta: \mathfrak{F}^{a b} \rightarrow C$ such that $f_{i, v} \mapsto \varsigma_{i}(v)$ and this is an isomorphism onto $C$ with inverse given by $\varsigma_{i}(v) \mapsto f_{i, v}$ by Prop 10.5 and Cor. 9.3 We have proven the following

Proposition 10.6. The algebra $\mathfrak{F}^{a b}$ is a free polynomial ring freely generated by $f_{i, v}$ with $i \geq 1$ and $v$ that varies in a complete set of representatives of $\Psi$.

Remark 10.7. The above Proposition can be also proved observing that $\mathfrak{F} \cong \hat{\Gamma}\left(F^{+}\right)$ where the latter has been introduced by Ziplies [18] and applying then Th.4.4 19.

10.4. A presentation. The assignment $R \mapsto R^{a b}$ gives a functor from $\mathbb{K}$-algebras to commutative $\mathbb{K}$-algebras. It preserves surjections and for $\rho: R \rightarrow S$ we denote by $\tilde{\rho}: R^{a b} \rightarrow S^{a b}$ its image by $a b$. Recall that we denote by $\mathfrak{a b}: R \rightarrow R^{a b}$ the canonical projection. We finally state the main result of this section

Theorem 10.8. Let $\mathbb{K}$ be a commutative ring and let $F$ be a free $\mathbb{K}$-algebra then

$$
0 \longrightarrow\left(\mathfrak{F}^{n}\right)^{a b} \longrightarrow(\mathfrak{F})^{a b} \stackrel{\tilde{\sigma}_{n}}{\longrightarrow} T S_{\mathbb{K}}^{n} F^{a b} \longrightarrow 0
$$

is a presentation by generators and relations. 
Corollary 10.9. Let $R \cong F / I$ be a $\mathbb{K}$-algebra generated by $\left\{r_{j}\right\}$ then we have a presentation by generators and relations

$$
0 \longrightarrow\left(\mathfrak{F}^{n}\right)^{a b}+J \longrightarrow(\mathfrak{F})^{a b} \longrightarrow \Gamma_{\mathbb{K}}^{n}(R)^{a b} \longrightarrow 0
$$

where $J$ is linearly generated by the lifting of $e_{\alpha}^{n}\left(f_{1}, \ldots, f_{k}\right)$ where at least one $f_{h} \in I$.

Proof. By [2] es.2)(f) pag.92.

Remark 10.10. the above corollary gives generators and relations for $\Gamma_{\mathbb{K}}^{n}(R)$ for any commutative ring $R$.

Corollary 10.11. Let $\mathbb{K}$ be an infinite field then $\mathfrak{F}^{n}$ and $\left(\mathfrak{F}^{n}\right)^{a b}$ are generated as ideals by the lifting of the $e_{n+k}^{h}(f)$ with $f \in F$ and their images by $\mathfrak{a b}$ respectively.

Proof. Over an infinite field one has that $T S_{\mathbb{K}}^{n}(M)$ is linearly generated by $m^{\otimes n}$ as $m \in M$ for any $M \in M o d_{\mathbb{K}}$.

\section{INVARIANTS}

The general linear group $G:=G L(n, \mathbb{K})$ made of the invertible matrices of $\operatorname{Mat}(n, \mathbb{K})$ acts on $\operatorname{Mat}(n, \mathbb{K})^{m}$ by simultaneous conjugation, i.e. via base change on $\mathbb{K}^{n}$. Recall that the categorical quotient $\mathcal{M}_{n}^{m} / / G$ is defined as

$$
\mathcal{M}_{n}^{m} / / G:=\operatorname{Spec}\left(A^{G}\right)
$$

where as usual $A_{n}^{G}$ denotes the ring of the invariants, $\mathcal{M}_{n}^{m}=\operatorname{Spec}\left(A_{n}^{G}\right)$ and the action of $G$ on $A_{n}$ is induced by the one on $\operatorname{Mat}(n, \mathbb{K})^{m}$. This scheme has be widely studied and we refer the reader to 1, 5, 8 for masterpieces on this subject. It is the coarse moduli space parameterizing the $n$-dimensional linear representations of $F$ up to base change.

Being the determinant invariant under base change we have that the ring $C_{n}$ is made of invariants i.e $C_{n} \subset A_{n}^{G}$. When $\mathbb{K}$ is a characteristic zero field was showed firstly by C. Procesi [8] and separately Sibirsky that $C_{n}=A_{n}^{G}$. This has been generalized to the positive characteristic case by S. Donkin and then by A. Zubkov proving a Procesi's conjecture, see [5, 8, 20. It also prove that the result remain true over the integers showing indeed that $C_{n}$ is a $\mathbb{Z}$ form of the ring of invariants, i.e. $\mathbb{K} \otimes_{\mathbb{Z}} C_{n} \cong \mathbb{K}\left[\operatorname{Mat}(n, \mathbb{K})^{m}\right]^{G L(n, \mathbb{K})}$. Zubkov in [21, gave a generalization of the well celebrated Procesi-Razmyslov theorem 91 10, namely he proved that the kernel of the surjection $C \rightarrow C_{n} \cong A_{n}^{G}$ is generated by $\psi_{n+k}(f)$ with $k \geq 1$ when $\mathbb{K}$ is an infinite field.

Combining these results on the invariants of matrices with Theorem 10.8 and Corollary 10.11 we finally have

Theorem 11.1. Let $\mathbb{K}$ be an infinite field or the ring $\mathbb{Z}$ of integers. The homomorphism $T S_{\mathbb{K}}^{n}(F)^{a b} \rightarrow \mathbb{K}[M a t(n, \mathbb{K})]^{G L(n, \mathbb{K})}$ induced by the composition det $\cdot \pi_{n}$ of the determinant with the universal representation is an isomorphism

$$
T S_{\mathbb{K}}^{n}(F)^{a b} \stackrel{\cong}{\rightarrow} \mathbb{K}[\operatorname{Mat}(n, \mathbb{K})]^{G L(n, \mathbb{K})}
$$


Proof. When $\mathbb{K}$ is an infinite field it follows by the results on the invariants of matrices recalled above, Theorem 10.8 and Corollary 10.11 It remains to prove only the case of $\mathbb{K}=\mathbb{Z}$. For any commutative ring $\mathbb{K}$ one has $T S_{\mathbb{K}}^{n}(F) \cong \mathbb{K} \otimes_{\mathbb{Z}} T S_{\mathbb{Z}}^{n}(F)$ then the homomorphism $i d_{\mathbb{K}} \otimes \mathfrak{a b}_{\mathbb{Z}}: \mathbb{K} \otimes_{\mathbb{Z}} T S_{\mathbb{Z}}^{n}(F) \rightarrow \mathbb{K} \otimes_{\mathbb{Z}} T S_{\mathbb{Z}}^{n}(F)^{a b}$ factors through $\mathfrak{a b}_{\mathbb{K}}: T S_{\mathbb{K}}^{n}(F) \rightarrow T S_{\mathbb{K}}^{n}(F)^{a b}$ by universality of $a b$ and the following diagram commutes

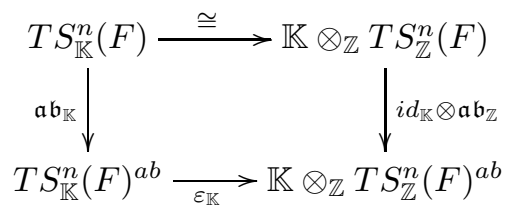

Let $\mathbb{K}$ be an algebraically closed field.

We have a map $T S_{\mathbb{Z}}^{n}(F)^{a b} \rightarrow \mathbb{Z}\left[M a t(n, \mathbb{Z})^{m}\right]^{G L(n, \mathbb{Z})}$ induced by the composition of the universal $n$-dimensional representation with the determinant, this gives another one $\mathbb{K} \otimes_{\mathbb{Z}} T S_{\mathbb{Z}}^{n}(F)^{a b} \rightarrow \mathbb{K} \otimes_{\mathbb{Z}} \mathbb{Z}\left[\operatorname{Mat}(n, \mathbb{Z})^{m}\right]^{G L(n, \mathbb{Z})}$ that can be lifted to

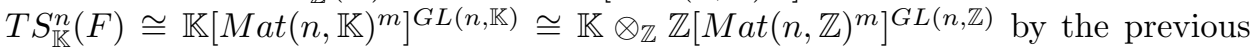
discussion and $\left[\underline{5}\right.$. Compl. We have then $\mathbb{K} \otimes_{\mathbb{Z}} T S_{\mathbb{Z}}^{n}(F)^{a b} \cong \mathbb{K} \otimes_{\mathbb{Z}} \mathbb{Z}\left[M a t(n, \mathbb{Z})^{m}\right]^{G L(n, \mathbb{Z})}$ for any algebraically closed field as $\mathbb{N}^{m}$-graded rings with finitely generated homogenous summand and the result follows.

\section{ACKNOWLEDGEMENTS}

I would like to thank C. Procesi, M. Brion and C. De Concini for hints and useful discussions. A special thank to David Rydh for valuable comments on the first version of this paper.

\section{REFERENCES}

[1] Artin, M.: On Azumaya algebras and finite-dimensional representations of rings. J.Algebra 11, 532-563 (1969)

[2] Bourbaki, N.: Elements of mathematics - Algebra II Chapters 4-7. Springer-Verlag, Berlin (1988)

[3] De Concini, C., Procesi, C., Reshetikhin, N., Rosso, M.: Hopf algebras with trace and representations. Invent. Math. 161, 1-44 (2005)

[4] Deligne, P.: Cohomologie á support propres. Théories des Topos et Cohomologie Étale des Schemas, Tome 3, Séminaire de Géometrie Algébrique du Bois Marie 1963/64, SGA 4. LNM 305, 250-480 (1973)

[5] Donkin, S.: Invariants of several matrices. Invent. Math. 110, 389-401 (1992)

[6] Donkin, S.: Invariant functions on matrices. Math. Proc. Cambridge Philos. Soc. 113, (1993) 23-43

[7] Macdonald, I.G.: Symmetric Functions and Hall Polynomials - second edition. Oxford mathematical monograph, Oxford (1995)

[8] Procesi, C.: Non-commutative affine rings. Atti Accad. Naz. Lincei Mem. Cl. Sci. Fis. Mat. Natur. Sez. I 8 237-255 (1967)

[9] Procesi, C.: The invariant theory of $n \times n$ matrices. Adv.Math. 19 306-381 (1976)

[10] Razmyslov, Ju. P.: Identities with trace in full matrix algebras over a field of characteristic zero. Izv. Akad. Nauk SSSR Ser. Mat. 38 723-756 (1974)

[11] Rydh, D.: A minimal set of generators for the ring of the multisymmetric functions. Preprint (2006).

[12] Roby, N.: Lois polynômes et lois formelles en théorie des modules. Ann.Ecole Norm. Sup. 80 213-348 (1963)

[13] Roby, N.: Lois polynômes multiplicatives universelles. C. R. Math. Acad. Sci. Paris 290 869-871 (1980) 
[14] Vaccarino, F.: On the Invariants of Matrices and the Embedding Problem. math.RA0406204 (2004)

[15] Vaccarino, F.: The ring of multisymmetric functions. Ann. Inst. Fourier 55 717-731 (2005)

[16] Vaccarino, F.: Linear representations, symmetric products and the commuting scheme. Submitted for publication. math.AG/0602660 (2006)

[17] Vaccarino, F. : The Hilbert-Chow morphism, symmetric product and the commuting variety. Short Communication ICM 2006, Madrid

[18] Ziplies, D. Generators for the divided powers algebra of an algebra and trace identities. Beiträge Algebra Geom. 24 9-27 (1987)

[19] Ziplies, D.: Abelianizing the divided powers algebra of an algebra. J.Algebra 122 261-274 (1989)

[20] Zubkov, A.N.: Endomorphisms of tensor products of exterior powers and Procesi hypothesis. Comm.Algebra 22 6385-6399 (1994)

[21] Zubkov, A.N.: On a generalization of the Razmyslov-Procesi theorem. Algebra and Logic 35 241-254 (1996)

Politecnico di Torino - DispeA, Corso Duca degli Abruzzi 24, I-10129, Torino, Italy

E-mail address: francesco.vaccarino@polito.it 